\title{
Capsule Commentary on Singh et al., A Qualitative Evaluation of Geographical Localization of Hospitalists: How Unintended Consequences May Impact Quality
}

\author{
Shuhei Hattori, M.D.
}

Rakuwakai Otowa Hospital, Kyoto, Japan.

J Gen Intern Med 29(7): 1056

DOI: $10.1007 / \mathrm{s} 11606-014-2837-6$

(c) Society of General Internal Medicine 2014

$\mathrm{T}$ his study qualitatively assessed the impact of colocating hospitalists and nurses on patient care. ${ }^{1}$ Using focus groups, nurse and provider participants reported a positive impact, mediated by proximity and improved communication. Focus groups also developed a conceptual model demonstrating how geographic localization could affect several key elements of patient care.

This paper also elucidated four potential unintended consequences of co-localization: 1) increased communication interruptions, which could threaten patient safety; 2) rather than a constant flow of new patients from multiple teams, co-located teams had admission flow problems, with patients often arriving in boluses, which tended to overwhelm nursing resources; 3) the teams were generalist teams and this could potentially disrupt wards with nursing units that are specialized; and 4) there was the potential for perverse incentives to increase length of stay, as found in previous work. $^{2}$

This study has several limitations. The sample size was small, including only two hospitalist teams and one nursing unit, and many constituents of medical teams, such as pharmacists, case managers and social workers, were not included. Moreover, despite the primary interest in the impact on patient care, there were no patients' voices reflected in the results. Despite these limitations, the model looks reasonable and fits well with what we imagine would happen if this co-localization were deployed. For the next step, researchers might want to further refine their conceptual model by more qualitative research with a larger, more diverse sample including staff other than doctors and nurses, incorporating the patient's perspective.

In addition, further quantitative investigations on the impact of co-localization are also needed. Previous research of co-localization of hospitalist with nursing teams found increased satisfaction and no impact on readmission rates, ${ }^{2,3}$ but no improvement in agreement between nurses and providers on the care plan $^{2}$ and possibly increased length of stay. ${ }^{3}$ Research on uninvestigated domains of quality such as patient safety is also a future task.

Finally, ward teams provide functions other than patient care; for example, education. The potential impact of colocalization on aspects of the medical enterprise other than direct patient care should be evaluated.

Conflict of Interest: The author has no conflicts of interest with this article.

Corresponding Author: Shuhei Hattori, M.D.; Rakuwakai Otowa Hospital, Kyoto, Japan (e-mail: Nin.nin810@gmail.com).

\section{REFERENCES}

1. Singh S, Fletcher KE. A qualitative evaluation of geographical localization of hospitalists: How unintended consequences may impact quality. J Gen Intern Med DOI: 10.1007/s11606-014-2780-14.

2. Singh S, Tarima S, Rana V, et al. Impact of localizing general medical teams to a single nursing unit. J Hosp Med. 2012;7(7):551-556.

3. O'Leary K, Wayne D, Landler M, et al. Impact of Localizing Physicians to Hospital Units on Nurse-Physician Communication and Agreement on the Plan of Care. J Gen Intern Med. 2009;24(11):1223-1227. 